\title{
Patients with Poor Response to Antipsychotics Have a More Severe Pattern of Frontal Atrophy: A Voxel-Based Morphometry Study of Treatment Resistance in Schizophrenia
}

\author{
Mario Quarantelli, ${ }^{1}$ Olga Palladino, ${ }^{2}$ Anna Prinster, ${ }^{1}$ Vittorio Schiavone, ${ }^{3}$ \\ Barbara Carotenuto, ${ }^{4}$ Arturo Brunetti, ${ }^{4}$ Angela Marsili, ${ }^{5}$ Margherita Casiello, ${ }^{2}$ \\ Giovanni Muscettola, ${ }^{2}$ Marco Salvatore, ${ }^{4}$ and Andrea de Bartolomeis ${ }^{2}$ \\ ${ }^{1}$ Biostructure and Bioimaging Institute, National Research Council, Via T. De Amicis 95, 80145 Naples, Italy \\ ${ }^{2}$ Section of Psychiatry, Department of Neuroscience and Reproductive and Odontostomatological Sciences, \\ University "Federico II", 80131 Naples, Italy \\ ${ }^{3}$ IDC Hermitage Capodimonte Spa, 80145 Naples, Italy \\ ${ }^{4}$ Department of Advanced Biomedical Sciences, University "Federico II", 80131 Naples, Italy \\ ${ }^{5}$ Department of Neuroscience, Reproductive and Odontostomatological Sciences, University "Federico II", 80131 Naples, Italy
}

Correspondence should be addressed to Mario Quarantelli; quarante@unina.it

Received 13 February 2014; Revised 20 June 2014; Accepted 11 July 2014; Published 23 July 2014

Academic Editor: Annalena Venneri

Copyright (C) 2014 Mario Quarantelli et al. This is an open access article distributed under the Creative Commons Attribution License, which permits unrestricted use, distribution, and reproduction in any medium, provided the original work is properly cited.

\begin{abstract}
Approximately $30 \%$ of schizophrenia patients do not respond adequately to the therapy. Previous MRI studies have suggested that drug treatment resistance is associated with brain morphological abnormalities, although region-of-interest analysis of MR studies from nonresponder and responder patients failed to demonstrate a statistically significant difference between these two schizophrenia subgroups. We have used a voxel-based analysis of segmented MR studies to assess structural cerebral differences in 20 nonresponder and 15 responder patients and 16 age-matched normal volunteers. Differences between the three groups emerged bilaterally mainly at the level of the superior and middle frontal gyri, primarily due to reduced grey matter volumes in nonresponders, as compared to both normal volunteers and responder patients. Post hoc direct comparison between the two schizophrenia subgroups demonstrated significantly reduced grey matter volumes in middle frontal gyrus bilaterally, in the dorsolateral aspects of left superior frontal gyrus extending into postcentral gyrus and in the right medial temporal cortex. Our results extend and integrate previous findings suggesting a more severe atrophy in nonresponder schizophrenia patients, compared to responder patients, mainly at the level of the superior and middle frontal gyri. Longitudinal studies in drug-naïve patients are needed to assess the role of these associations.
\end{abstract}

\section{Introduction}

Antipsychotics represent the mainstay of schizophrenia pharmacological treatment; however, approximately $20 \%$ to $35 \%$ of people affected by schizophrenia, under optimal antipsychotic treatment and when all major cofactors are controlled for, fail to respond to antipsychotics [1-6]. Treatmentresistant schizophrenia patients show a reduced quality of life, increased drug abuse [7], and reduced cognitive performance compared to patients responders to the treatment [8].
Understanding the mechanisms of treatment response is relevant to schizophrenia pathophysiology and to the therapeutic implications. However, treatment-resistant schizophrenia, since its initial definition introduced by Kane and collaborators in the Multicenter Clozapine Trial [9], remains a post hoc diagnosis based on the clinical course. Clinical features at diagnosis such as poorer premorbid function, an earlier age at onset of positive symptoms, male gender, family history of schizophrenia, longer duration of untreated psychosis, severe negative symptoms, presence 
of soft neurological signs, absence of precipitating factors, and a history of substance abuse can only partially predict resistance to the treatment [10-13].

Although it has been speculated that brain imaging, both structural and functional, could contribute to the identification of biological variables related to treatment response or resistance, to the best of our knowledge there are only a few imaging studies on the putative structural correlates of drug-resistance [14-22], which overall suggest that a more severe pattern of brain alterations may underlie treatment resistance.

In this study, we tested the hypothesis that brain regional abnormalities may correlate with treatment resistance in schizophrenia patients. We applied a voxel-based analysis of segmented MRI images (brain-wise analysis, not based on predefined ROIs) to assess brain structural differences between NonResp-SC and Resp-SC patients and compare both groups with normal control.

\section{Material and Methods}

2.1. Subjects. Fifty-one subjects were studied. Fifteen RespSC and twenty NonResp-SC male patients were sequentially enrolled among the patient population referring to the Psychiatry Section, Unit of Treatment Resistant Psychosis at the Department of Neuroscience of the University of Naples Federico II.

The patients were referred to the unit by psychiatrists of Community Health Centers, private practice psychiatrists or by general physicians. All the NonResp-SC patients had been already treated with at least two antipsychotic trials when first admitted at Outpatient Clinics for treatment-resistant psychosis.

Exclusion criteria for patients were left-handedness; age below 18 years; evidence of cardiovascular, metabolic, or neurological impairment; previous head injuries requiring hospitalization; history of mental retardation, alcoholism, substance dependence over the previous 3 years or abuse over the previous 6 months; head injury or electroconvulsive therapy; and lack of willingness to participate to the study.

Sixteen age-matched male normal volunteers (NV) were also enrolled over the same timeframe through local advertising. Exclusion criteria for NV were evidence of cardiovascular, metabolic, neurological, and psychiatric impairment; previous head injuries requiring hospitalization; alcohol or recreational drugs use; or treatment with medications active on the CNS.

All patients underwent clinical assessment to confirm the diagnosis of schizophrenia according to DSM IV TR. The assessment was carried out by a psychiatrist (MC) with more than 20 years of experience in psychosis diagnosis and treatment and was confirmed by a second psychiatrist (GM) by means of a Structured Treatment resistant Record Chart, specifically developed at Unit of Treatment Resistant Psychosis.

The Expanded Brief Psychiatric Rating Scale (E-BPRS [25]) was administered to the patients on the same day of the MRI study.
Clinical data were collected from clinical records of the Outpatient Clinics for Drug Resistance at the Department of Neuroscience, including also patient family interviews, and the duration of psychosis (disease duration-DD) and the duration of untreated psychosis were calculated from the first manifestation of psychotic symptoms such as delusions, hallucinations, thought disorder, or inappropriate/bizarre behavior lasting throughout the day for several days or several times a week and requiring an unambiguous psychiatric intervention [26].

Patients were classified as NonResp-SC based on the lack of a satisfactory clinical improvement despite the sequential use of the recommended doses for 6 to 8 weeks of at least two antipsychotics where at least one of which is atypical [27].

Lack of clinical improvement was defined by all of the following conditions:

(1) lack of $\geq 20 \%$ improvement and persistence of a value $\geq 45$ in the total score of the 18 -items version of the BPRS (included in the E-BPRS) [9];

(2) a score $\geq 4$ in at least two out of the four E-BPRS psychotic items.

In addition to the above criteria, the presence of poor psychosocial and community functioning, which persisted for at least two years despite trials of medication that have been adequate in terms of dose, duration, and adherence was required [28].

The compliance was ascertained for each patient according to the following check points [29]:

(1) clinical records of previous psychiatric assessments (either as outpatient or as inpatient)

(2) interview with patient and family member or caregivers (that was systematically available for all patients enrolled in the study) addressing systematically the following issues:

(a) timing of therapy administration;

(b) How many times in a week the medicine was eventually missed (we excluded patients who missed the medicine more than seven times in one month or for three days in row for more than twice in the last month before the assessment);

(c) Attitude of the patient toward the medicine: expectation toward disease control, acceptance of potential or actual side effects, and willing to take the therapy after resolution of acute psychotic episode;

(d) Co-occurrence of prolonged other medicines administration that could influence, by pharmacokinetic interference, the efficacy of antipsychotics (i.e., polytherapy such as carbamazepine plus haloperidol) and could be responsible for symptoms rebound.

All enrolled patients were under antipsychotic treatment at the time of the MRI scan. Current treatments included 
TABle 1: Demographic and clinical data.

\begin{tabular}{|c|c|c|c|c|c|c|c|c|}
\hline & & Age & $\mathrm{DD}$ & $\mathrm{AAO}$ & Antipsychotics* & E-BPRS & BPRS-PS & BPRS-NS \\
\hline \multirow{5}{*}{$\begin{array}{l}\mathrm{NV} \\
(n=16)\end{array}$} & Mean & 35.2 & $\mathrm{n} / \mathrm{a}$ & $\mathrm{n} / \mathrm{a}$ & $\mathrm{n} / \mathrm{a}$ & $\mathrm{n} / \mathrm{a}$ & $\mathrm{n} / \mathrm{a}$ & $\mathrm{n} / \mathrm{a}$ \\
\hline & SD & 11.3 & $\mathrm{n} / \mathrm{a}$ & $\mathrm{n} / \mathrm{a}$ & $\mathrm{n} / \mathrm{a}$ & $\mathrm{n} / \mathrm{a}$ & $\mathrm{n} / \mathrm{a}$ & $\mathrm{n} / \mathrm{a}$ \\
\hline & Median & 35.8 & $\mathrm{n} / \mathrm{a}$ & $\mathrm{n} / \mathrm{a}$ & $\mathrm{n} / \mathrm{a}$ & $\mathrm{n} / \mathrm{a}$ & $\mathrm{n} / \mathrm{a}$ & $\mathrm{n} / \mathrm{a}$ \\
\hline & Min. & 21.1 & $\mathrm{n} / \mathrm{a}$ & $\mathrm{n} / \mathrm{a}$ & $\mathrm{n} / \mathrm{a}$ & $\mathrm{n} / \mathrm{a}$ & $\mathrm{n} / \mathrm{a}$ & $\mathrm{n} / \mathrm{a}$ \\
\hline & Max. & 57.0 & $\mathrm{n} / \mathrm{a}$ & $\mathrm{n} / \mathrm{a}$ & $\mathrm{n} / \mathrm{a}$ & $\mathrm{n} / \mathrm{a}$ & $\mathrm{n} / \mathrm{a}$ & $\mathrm{n} / \mathrm{a}$ \\
\hline \multirow{5}{*}{$\begin{array}{l}\text { Resp-SC } \\
(n=15)\end{array}$} & Mean & 33.5 & 11.1 & 22.3 & $310.6^{\S}$ & $26.9^{\S}$ & $5.7^{\S}$ & $3.3^{\S}$ \\
\hline & $\mathrm{SD}$ & 11.2 & 9.6 & 9.4 & 300 & 3.8 & 1.2 & 0.6 \\
\hline & Median & 28.8 & 8 & 20.2 & 239.9 & 25 & 5 & 3 \\
\hline & Min. & 20.5 & 1 & 12.5 & 60 & 24 & 5 & 3 \\
\hline & Max. & 54.6 & 30 & 48.5 & 1008 & 35 & 9 & 5 \\
\hline \multirow{5}{*}{$\begin{array}{l}\text { NonResp-SC } \\
(n=20)\end{array}$} & Mean & 33.1 & 13.0 & 20.1 & 591.4 & 73.3 & 23.05 & 8.55 \\
\hline & SD & 7.6 & 6.8 & 3.2 & 525 & 9.2 & 4.3 & 3.3 \\
\hline & Median & 33.8 & 13 & 20.1 & 331.3 & 76.5 & 24.5 & 8.5 \\
\hline & Min. & 19.2 & 1 & 14.2 & 120 & 49 & 11 & 4 \\
\hline & Max. & 51.7 & 30 & 26.6 & 1350 & 86 & 28 & 17 \\
\hline
\end{tabular}

DD: Disease Duration (years).

AAO: Age at onset (years).

E-BPRS: Expanded Brief Psychiatric Rating Scale, version 4.0.

BPRS-PS: BPRS Positive Symptoms.

BPRS-NS: BPRS Negative Symptoms.

n/a: not applicable.

${ }^{*}$ Current daily dose of antipsychotics converted in mg equivalents of chlorpromazine [23].

${ }^{\S}$ Significatly different between Resp-SC and NonResp-SC $(P<0.005$ at Mann-Whitney test).

For E-BPRS BPRS-PS, and BPRS-NS (higher scores indicate more severe psychiatric symptoms).

haloperidol, olanzapine, aripiprazole, bromperidol, and risperidone in Resp-SC and haloperidol, olanzapine, clozapine, quetiapine, paliperidone, risperidone, clotiapine, and perfenazine in NonResp-SC. The total daily doses of antipsychotics, converted in chlorpromazine mg equivalents per day [23], reported in Table 1, were significantly higher in NonResp-SC patients ( $P=0.003$ at Mann-Whitney test).

Concomitant treatments included selective serotonin reuptake inhibitors (in 1 Resp-SC and 4 NonResp-SC), benzodiazepines (in 3 Resp-SC and 10 NonResp-SC), antiparkinsonian drugs (in 2 Resp-SC and 4 NonResp-SC), and anticonvulsants (in 5 NonResp-SC).

Demographic and clinical data of patient and NV groups are summarized in Table 1 . No significant difference was present at ANOVA among the three groups (NV, Resp-SC, and NonResp-SC) in terms of age or between the two SC subgroups in terms of DD and age at onset (AAO).

The two SC subgroups were significantly different in terms of total E-BPRS score $\left(P<10^{-9}\right.$ at MannWhitney test), with significantly higher scores in the NonResp-SC group; this difference was significant also when assessing separately the E-BPRS subscores related to positive psychotic symptoms (BPRS-PS comprising the hallucinatory behavior, unusual thought content, suspiciousness, and conceptual disorganization $[30,31]$ and mannerism items [32]; $P<10^{-8}$ ) and negative symptoms (BPRS-NS comprising the blunted affect, emotional withdrawal and motor retardation items [32-34]; $P<$ $\left.10^{-7}\right)$.
The work was carried out in accordance with The Code of Ethics of the World Medical Association (Declaration of Helsinki) for experiments involving humans. All participating subjects gave written consent after the purpose and methods of the study had been explained to them and/or to their legal representatives, and the ethical committees of the participating institutions approved the protocol.

2.2. MR Studies and Segmentation. For segmentation purposes, T1-weighted volumes were acquired at 1.5 Tesla (Achieva, Philips Medical Systems, Eindhoven, The Netherland) using a magnetization-prepared 3D fast Gradient-Echo sequence $(\mathrm{TR} / \mathrm{TE} / \mathrm{TI} 11 / 2 / 600 \mathrm{~ms}$, voxel size $0.98 \times 0.98 \times$ $1.2 \mathrm{~mm}, 124$ contiguous axial slices covering the entire brain).

All scans were performed on the same MR scanner, and no HW/SW upgrade was carried out on the scanner during the study.

SPM5 (Wellcome Department of Cognitive Neurology, London, UK, http://www.fil.ion.ucl.ac.uk/spm/software/ spm5/) was used to segment the T1-weighted volumes into GM, WM, and CSF probabilistic maps, using the unified segmentation approach [35], which is a fully automated procedure combining within a single framework bias correction, template registration, and tissue classification, thus obviating the need for iterative steps including generation of a site-and study-specific MRI template.

2.3. Statistical Analysis: Global Tissues. Following segmentation, for each study, the GM, WM, and CSF volumes were 
calculated (for each tissue, as the sum of the corresponding probabilities multiplied by voxel volume, only in voxels where the cumulated probabilities of GM, WM and CSF exceeded $50 \%)$.

Differences in global brain tissue volumes among the three groups were assessed by general linear model multivariate analysis including total intracranial volume (ICV, the sum of GM, WM, and CSF volumes) and age in the model. Subsequently, only tissues that exhibited a significant difference among groups at MANOVA were tested by three post hoc linear regression analyses of significant main effects (NV versus NonResp-SC, NV versus Resp-SC, and Resp-SC versus NonResp-SC), to localize differences among groups.

2.4. Morphometric Analysis. For subsequent voxel-based analysis, GM volumes were normalized to the Montreal Neurological Institute (MNI) space using the SPM mean GM template with 16 nonlinear iterations using $6 \times 8 \times 6$ basis functions to account for global shape differences [36]. Normalized images were resampled by trilinear interpolation to $2 \times 2 \times 2 \mathrm{~mm}$ voxel size. To ensure that the total amount of GM in each region remained unchanged after the warping inherent to spatial normalization, thus allowing subsequent testing for voxelwise differences in the relative volume of GM [37], modulation of normalized GM maps was performed by multiplying the voxel values by the Jacobians derived from the corresponding spatial normalization parameters [38].

Finally, modulated volumes were smoothed with a $5 \mathrm{~mm}$ FWHM 3D isotropic Gaussian filter. Local differences in gray matter volume between the three groups were assessed using permutation tests $[39,40]$ implemented in the Cambridge Brain Analysis software (CamBA version 2.3.0; http://www.bmu.psychiatry.cam.ac.uk/software) running under Linux.

This nonparametric method of analysis allows us to test the null hypothesis of no differences in regional brain tissue volumes between different groups, at the level of spatially contiguous $3 \mathrm{D}$ voxel clusters, thus incorporating spatial information, and has been shown to be generally more powerful than other tests, such as those informed only by data at the single voxel level [39-41].

Accordingly, an analysis of covariance (ANCOVA) model was fitted for each intracerebral voxel in the standard space, included in the model as covariates age and ICV. A preliminary voxelwise omnibus ANCOVA, including ICV and age as covariates, was performed to localize relative GM group differences among the three groups. Subsequently, three post hoc analyses of significant main effects (NV versus Resp-SC, NV versus NonResp-SC, and Resp-SC versus NonResp-SC) were performed, restricted to voxels significantly different among the three groups at the omnibus test.

For each post hoc test both direct and inverse contrasts were probed.

For each analysis, a preliminary probability threshold $(P<0.05)$ was applied to the corresponding voxel statistic maps and subthreshold voxels were set to 0 , thus creating a set of suprathreshold voxel clusters. The sum of the suprathreshold voxel values (cluster mass, $M$ ) was then tested against

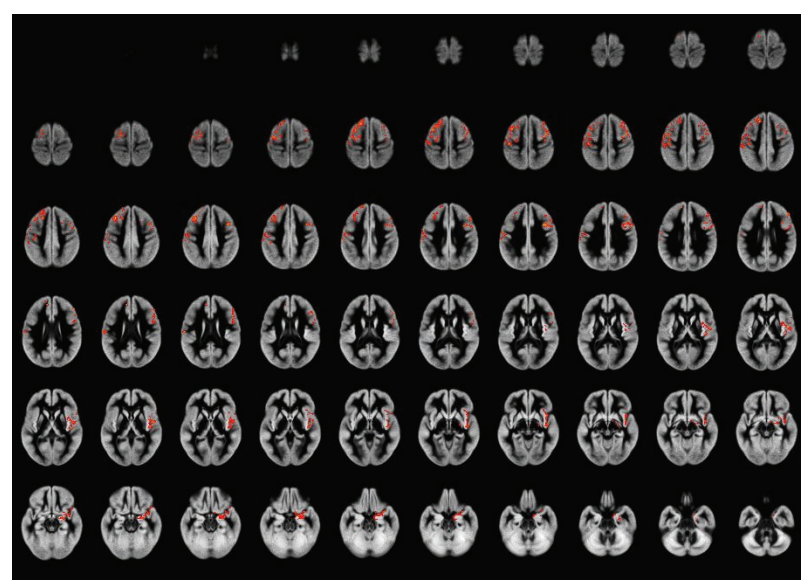

FIGURE 1: Areas of significant gray matter differences (including correction for age and total intracranial volume, less than 1 falsepositive cluster expected, $P<0.001$ ) across the three groups (normal volunteers and responder and nonresponder schizophrenia patients) at ANCOVA, superimposed onto the average of the normalized GM volumes. Right side of the brain is at the observer's right. GM differences among the three groups involve mainly the frontal lobes bilaterally. Superior and middle frontal gyri are involved on the left, extending into the pre- and postcentral gyri, while on the right mainly middle frontal gyrus and insula are involved. Additionally, right medial temporal lobe (mainly amygdala) is involved.

the $\mathrm{M}$ distribution obtained from 10 random permutations of the data sets. Probability thresholds for cluster testing were then set so that the average number of false-positive clusters expected per map was less than one [39, 40].

Clusters showing significant between group differences were localized based on their coordinates in the MNI space [24].

\section{Results}

3.1. Global Brain Tissue Volumes. Analysis of global brain tissue volumes showed a significant group effect at multivariate analysis $(P<0.05)$ for global GM volume, corrected for age and intracranial volume (ICV). Post hoc pairwise comparisons showed the effect on GM variance to be due to reduced GM volumes in the NonResp-SC $(P=0.03)$ compared to NV, while no significant differences emerged between Resp-SC and NV or between the two SC subgroups.

3.2. Morphometric Analysis. Clusters of significant GM differences among the three groups, as detected by morphometric analysis, are displayed in Figure 1. The results of the three post hoc comparisons (NV versus Resp-SC, NV versus NonResp-SC, and NonResp-SC versus Resp-SC) are shown in Figures 2, 3, and 4. Corresponding significant cluster size and locations are reported in Table 2.

Main differences between the three groups emerged bilaterally at the level of the superior and middle frontal gyri, with extension to the postcentral gyrus on the left and involvement of right insula and medial temporal cortex. 
TABLE 2: Regional gray matter differences across the three groups.

\begin{tabular}{|c|c|c|c|c|c|}
\hline \multirow{2}{*}{ Region } & \multirow{2}{*}{ Side } & \multirow{2}{*}{ Omnibus } & \multicolumn{3}{|c|}{ POST-HOC } \\
\hline & & & Resp-SC $<\mathrm{NV}$ & NonResp-SC $<\mathrm{NV}$ & NonResp-SC $<$ Resp-SC \\
\hline Postcentral gyrus & Left & 2.2 & & 2 & 0.8 \\
\hline Precentral gyrus & Left & 1.5 & 0.6 & 1.4 & \\
\hline Superior frontal gyrus, dorsolateral & Left & 2.4 & & 1.6 & 0.9 \\
\hline Middle frontal gyrus & Left & 3.1 & 0.6 & 2.9 & 1.5 \\
\hline Precentral gyrus & Right & 1 & & 0.8 & \\
\hline Rolandic operculum & Right & 0.5 & & 0.5 & \\
\hline Middle Frontal gyrus & Right & 2.2 & & 2.2 & 0.6 \\
\hline Inferior frontal gyrus, opercular part & Right & 1.1 & & 1 & \\
\hline Inferior frontal gyrus, triangular part & Right & 0.7 & & 0.6 & \\
\hline Insula & Right & 2.2 & & 2.2 & \\
\hline Amygdala & Right & 0.7 & & 0.7 & \\
\hline
\end{tabular}

Volumes (in cc) of the clusters of significant gray matter differences (less than 1 false-positive cluster expected) across the three groups of patients at ANCOVA (omnibus test) and at the three post-hoc tests.

Localization is according to Tzourio-Mazoyer, et al. [24].

Structures involved for less than $0.5 \mathrm{cc}$ are not listed.

NV: Normal Volunteers.

NonResp-SC: Non-Responder schizophrenia patients.

Resp-SC: Responder schizophrenia patients.

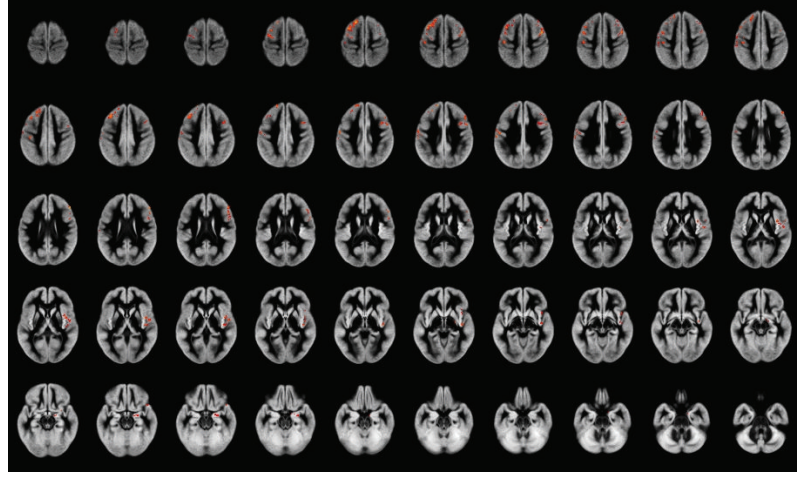

FIgURE 2: Areas of significant gray matter differences at ANCOVA (including correction for age and total intracranial volume, less than 1 false-positive cluster expected, $P<0.005$ ) between normal volunteers and nonresponder schizophrenia patients, superimposed onto the average of the normalized GM volumes. Right side of the brain is at the observer's right. GM reduction in nonresponder schizophrenia patients involves mainly the frontal lobes bilaterally. Superior and middle frontal gyri are involved on the left, extending into the pre- and postcentral gyri, while on the right mainly middle frontal gyrus and insula are involved. Additionally, right medial temporal lobe (mainly amygdala) is involved.

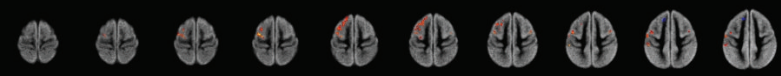

FIGURE 3: Areas of significant gray matter differences at ANCOVA (including correction for age and total intracranial volume, less than 1 false-positive cluster expected, $P<0.005)$ between normal volunteers and responder schizophrenia patients, superimposed onto the average of the normalized GM volumes. Right side of the brain is at the observer's right. Mainly left dorsal frontal cortex appears to be involved.

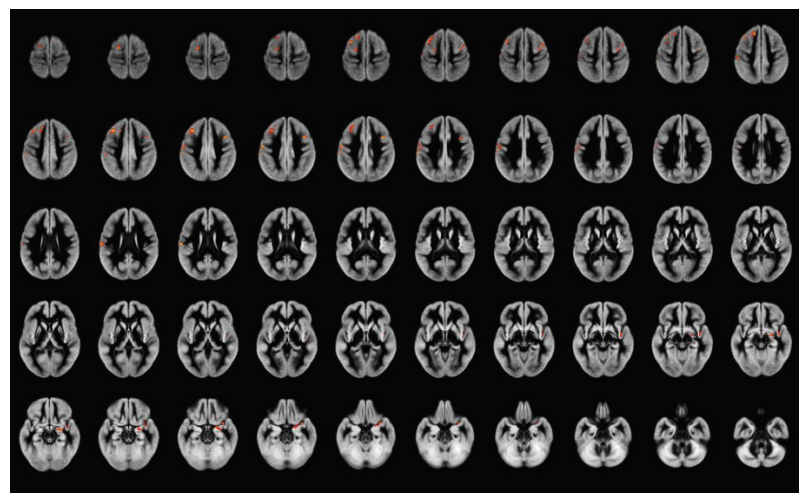

FIGURE 4: Areas of significant gray matter differences at ANCOVA (including correction for age and total intracranial volume, less than 1 false-positive cluster expected, $P<0.005)$ between responder and nonresponder schizophrenia patients, superimposed onto the average of the normalized GM volumes. Right side of the brain is at the observer's right. Clusters of significantly reduced GM volume in nonresponder schizophrenia patients compared to responder schizophrenia patients are detected mainly the frontal lobes bilaterally, more extended on the left where the pre- and postcentral gyri are involved, while on the right mainly middle frontal gyrus and insula are involved.

Post hoc analyses showed that these differences were due to a reduced GM volume of the frontal structures in NonResp-SC, compared to NV, while smaller clusters of significant GM loss were present in the R-CS compared to NV.

Finally, direct post hoc comparison of NonResp-SC and Resp-SC patients disclosed clusters of significantly reduced GM volume in NonResp-SC in superior and middle frontal gyri on the left, extending into postcentral cortex, and of 
middle frontal gyrus on the right, while smaller clusters (less than $0.5 \mathrm{cc}$ ) of reduced GM were detected in medial temporal lobe (mainly amygdala) and insula on the right.

No cluster of reduced GM volume was detected in NV versus both SC subgroups or in Resp-SC versus NonResp-SC.

\section{Discussion}

Our results demonstrate a significant reduction in GM volume in NonResp-SC mainly at the level of the superior and middle frontal gyri bilaterally and in right medial temporal cortex, as compared to Resp-SC.

Schizophrenic patients exhibit overall a decrease in brain volume in the temporal and frontal regions [42, 43]. A meta-analysis of gray matter anomalies in schizophrenia has shown that SC patients consistently show reduced gray matter density relative to control subjects in a network of regions, including bilateral insular cortices, anterior cingulate, left parahippocampal gyrus, left middle frontal gyrus, postcentral gyrus, and thalamus, coupled to increased gray matter density in striatal regions [44].

Previous neuroimaging studies using CT techniques have evaluated the correlation between frontal sulcal enlargement and poor clozapine response $[14,15,18]$.

MRI transversal studies of treatment resistance in schizophrenia have shown a trend to a greater atrophy in NonResp-SC when assessing global brain tissue volumes [17]. In a study, the patients who required higher-doses of haloperidol had greater deficits in gray matter volume than those who were treated with the lowest dose [22].

Subsequent efforts to localize regions of atrophy, as assessed by manual segmentation of MRI studies, which correlate with the response to treatment, have highlighted a possible role of right prefrontal cortex in the response to clozapine [21] (although in the same study larger right prefrontal grey matter volume was associated with poorer response to haloperidol).

More recently a study, directly assessing structural parameters in patients resistant to conventional antipsychotics using Region of Interest (ROI) analysis of segmented MRI [20], has shown that NonResp-SC patients have significantly lower gray matter (GM) volumes in the frontal and occipital regions and significantly more white matter (WM) in the frontal, parietal, and occipital regions as compared to the controls, while these alterations are less prominent in Resp-SC patients, although in that case the direct comparison between NonResp-SC and Resp-SC patients failed to reach statistical significance.

In addition, in a recent study of morphometric correlates of response to atypical neuroleptics a direct comparison of baseline MR studies of Resp-SC versus NonResp-SC, has suggested that higher GM volumes in left rectus gyrus volumes are associated with a better acute response to treatment with risperidone or olanzapine [45], although in that case this difference did not survive correction for multiple comparisons.

When comparing these results to ours, possible effects of different treatment regimens should be considered. Besides the well-known effects of typical antipsychotics on basalganglia volume, in fact, apparent increases in GM have also been detected in occipital and parietal cortex during clozapine treatment in NonResp-SC [46] in the inferior frontal cortex/orbitofrontal gyrus and anterior cingulate cortex during quetiapine treatment [47] and in the cingulate gyrus for typical agents [48].

The effect of different treatment should be taken into account as in our case the two SC subgroups were under different therapeutic regimens, which may partly explain discrepancies with previous results of ROI-based analysis of baseline studies in patients treated with haloperidol only, which highlighted differences in occipital GM, beside frontal cortex [20], while in our case the presence of a substantial proportion of NonResp-SC undergoing clozapine treatment may have hindered parietal and occipital differences with the Resp-SC subgroup.

The heterogeneity of antipsychotics in our patient population precluded however an analysis of drug-specific effects.

Although previous data have reported an association between GM atrophy and increased number of hospitalization [49] with poorer overall outcome [50], possibly suggesting that ongoing neurodegenerative phenomena may partly cause (or be related to) the development of treatment resistance [51], the possibility that the increased cortical atrophy, mainly in frontal regions, is a consequence of the treatment with antipsychotics, rather than being related to the causes of treatment resistance, should be also considered.

Although frontal atrophy is a feature consistent across schizophrenia subsyndromes [52], influenced by the genetic background [53], and detectable also in patients' relatives [54], it has been indeed suggested that it can be exacerbated by treatment with antipsychotic, an hypothesis supported to some extent by meta-analyses of MRI segmentation studies $[55,56]$ although with differences between typical and atypical ones $[49,57,58]$.

Furthermore, recent longitudinal studies in large cohorts of patients [58-60] have shown significant treatment effects on frontal GM atrophy, although without a significant treatment-by-time interaction [59], which would be expected if the cumulative effect of chronic therapy caused neurodegenerative phenomena.

Additionally, other factors should be considered when interpreting the meaning of structural differences in patient groups with a relatively large DD range, such as in our case. For example, differences of concurrent treatments between the two SC subgroups (antiepileptics and benzodiazepines being more frequently used in our NonResp-SC group of patients) may have also played a role in determining brain tissue volume differences, although the lack of a demonstrated persistent brain atrophy effect in chronic users of these classes of drugs $[61,62]$ mitigates against this hypothesis.

A limitation of the present study lies in the transversal approach in chronic patients, which precludes the possibility of extending the conclusions to first-episode patients, thus leaving open the question whether the more pronounced frontal GM loss is a cause or a consequence of the treatment resistance. 
In this respect it is of note that an MRI study in a large group of first-episode schizophrenia or schizoaffective disorder patients has not detected a significant effect of MRI variables onto prediction of treatment response [63], although in that case semiautomated ROI-based analysis of predefined brain structures, not covering the frontal lobes, was used.

Finally, for the purposes of the present study we dichotomized our patient population. However, the concept of treatment resistance may be recognized as a continuum rather than a dichotomy of response versus nonresponse [64], so that a correlation analysis in a larger cohort of patients could provide additional information.

\section{Conclusions}

We have shown significant structural cerebral differences in a direct comparison between two groups of NonRespSC and Resp-SC patients, suggesting an association between bilateral frontal and right medial temporal cortex alterations, and treatment resistance in schizophrenia. The results of the present study need to be confirmed in longitudinal studies in drug-naive, first-episode patients, to assess the role of these alterations and their possible predictive value.

\section{List of Abbreviations}

$\begin{array}{ll}\text { AAO: } & \text { Age at Onset } \\ \text { ANCOVA: } & \text { Analysis of covariance } \\ \text { BPRS-NS: } & \text { BPRS negative symptoms } \\ \text { BPRS-PS: } & \text { BPRS positive symptoms } \\ \text { CSF: } & \text { Cerebrospinal fluid } \\ \text { DD: } & \text { Disease duration } \\ \text { E-BPRS: } & \text { Expanded brief psychiatric rating scale } \\ \text { GM: } & \text { Gray matter } \\ \text { ICV: } & \text { Intracranial volume } \\ \text { MNI: } & \text { Montreal neurological institute } \\ \text { NonResp-SC: } & \text { Nonresponder schizophrenia } \\ \text { NV: } & \text { Normal volunteers } \\ \text { ROI: } & \text { Region of interest } \\ \text { Resp-SC: } & \text { Responder schizophrenia } \\ \text { SC: } & \text { Schizophrenia } \\ \text { WM: } & \text { White matter. }\end{array}$

\section{Conflict of Interests}

The authors declare that they have no conflict of interests.

\section{Authors' Contribution}

Mario Quarantelli participated in study design, carried out the MRI studies, and drafted the paper. Olga Palladino participated in the design of the study and in patient selection and evaluation. Anna Prinster carried out the morphometric analysis. Vittorio Schiavone participated in patient selection and evaluation. Barbara Carotenuto participated in study coordination and collaborated to the statistical analysis. Arturo Brunetti participated in study coordination and helped to draft the paper. Angela Marsili collaborated to MR studies acquisition and to the morphometric analysis. Margherita Casiello participated in patient selection and evaluation. Giovanni Muscettola participated in the design and coordination of the study. Marco Salvatore participated in the design and coordination of the study. Andrea de Bartolomeis conceived of the study and participated in its design and coordination. All authors read and approved the final paper.

\section{References}

[1] H. Elkis, "Treatment-resistant schizophrenia," Psychiatric Clinics of North America, vol. 30, no. 3, pp. 511-533, 2007.

[2] R. R. Conley and D. L. Kelly, "Management of treatment resistance in schizophrenia," Biological Psychiatry, vol. 50, no. 11, pp. 898-911, 2001.

[3] J. Lindenmayer, "Treatment refractory schizophrenia," Psychiatric Quarterly, vol. 71, no. 4, pp. 373-384, 2000.

[4] S. M. Essock, W. A. Hargreaves, N. H. Covell, and J. Goethe, "Clozapine's effectiveness for patients in state hospitals: results from a randomized trial," Psychopharmacology Bulletin, vol. 32, no. 4, pp. 683-697, 1996.

[5] H. D. Brenner, S. J. Dencker, M. J. Goldstein et al., "Defining treatment refractoriness in schizophrenia," Schizophrenia Bulletin, vol. 16, no. 4, pp. 551-561, 1990.

[6] R. R. Conley and R. W. Buchanan, "Evaluation of treatmentresistant schizophrenia," Schizophrenia Bulletin, vol. 23, no. 4, pp. 663-674, 1997.

[7] J. L. Kennedy, C. A. Altar, D. L. Taylor, I. Degtiar, and J. C. Hornberger, "The social and economic burden of treatmentresistant schizophrenia: a systematic literature review," International Clinical Psychopharmacology, vol. 29, no. 2, pp. 63-76, 2014.

[8] A. de Bartolomeis, R. Balletta, S. Giordano, E. F. Buonaguro, G. Latte, and F. Iasevoli, "Differential cognitive performances between schizophrenic responders and non-responders to antipsychotics: correlation with course of the illness, psychopathology, attitude to the treatment and antipsychotics doses," Psychiatry Research, vol. 210, no. 2, pp. 387-395, 2013.

[9] J. Kane, G. Honigfeld, J. Singer, and H. Meltzer, "Clozapine for the treatment-resistant schizophrenic: a double-blind comparison with chlorpromazine," Archives of General Psychiatry, vol. 45, no. 9, pp. 789-796, 1988.

[10] J. Lieberman, D. Jody, S. Geisler et al., "Time course and biologic correlates of treatment response in first-episode schizophrenia," Archives of General Psychiatry, vol. 50, no. 5, pp. 369-376, 1993.

[11] H. Y. Meltzer, J. Rabinowitz, M. A. Lee et al., "Age at onset and gender of schizophrenic patients in relation to neuroleptic resistance," The American Journal of Psychiatry, vol. 154, no. 4, pp. 475-482, 1997.

[12] E. C. Johnstone, D. G. C. Owens, G. M. Bydder, N. Colter, T. J. Crow, and C. D. Frith, "The spectrum of structural brain changes in schizophrenia: age of onset as a predictor of cognitive and clinical impairments and their cerebral correlates," Psychological Medicine, vol. 19, no. 1, pp. 91-103, 1989.

[13] T. Kolakowska, A. O. Williams, M. Ardern et al., "Schizophrenia with good and poor outcome. I: early clinical features, response to neuroleptics and signs of organic dysfunction," British Journal of Psychiatry, vol. 146, pp. 229-239, 1985. 
[14] L. Friedman, L. Knutson, M. Shurell, and H. Y. Meltzer, "Prefrontal sulcal prominence is inversely related to response to clozapine in schizophrenia," Biological Psychiatry, vol. 29, no. 9, pp. 865-877, 1991.

[15] W. G. Honer, G. N. Smith, J. S. Lapointe, G. W. MacEwan, L. Kopala, and S. Altman, "Regional cortical anatomy and clozapine response in refractory schizophrenia," Neuropsychopharmacology, vol. 13, no. 1, pp. 85-87, 1995.

[16] S. M. Lawrie, G. T. Ingle, C. G. Santosh et al., "Magnetic resonance imaging and single photon emission tomography in treatment-responsive and treatment-resistant schizophrenia," British Journal of Psychiatry, vol. 167, pp. 202-210, 1995.

[17] S. M. Lawrie, S. S. Abukmeil, A. Chiswick, V. Egan, C. G. Santosh, and J. J. K. Best, "Qualitative cerebral morphology in schizophrenia: A magnetic resonance imaging study and systematic literature review," Schizophrenia Research, vol. 25, no. 2, pp. 155-166, 1997.

[18] P. E. Konicki, K. Y. Kwon, V. Steele et al., "Prefrontal cortical sulcal widening associated with poor treatment response to clozapine," Schizophrenia Research, vol. 48, no. 2-3, pp. 173-176, 2001.

[19] H. A. Savas, B. Unal, H. Erbagci et al., "Hippocampal volume in schizophrenia and its relationship with risperidone treatment: a stereological study," Neuropsychobiology, vol. 46, no. 2, pp. 6166, 2002.

[20] V. Molina, S. Reig, J. Sanz et al., "Differential clinical, structural and P300 parameters in schizophrenia patients resistant to conventional neuroleptics," Progress in Neuro-Psychopharmacology \& Biological Psychiatry, vol. 32, no. 1, pp. 257-266, 2008.

[21] C. Arango, A. Breier, R. McMahon, W. T. Carpenter Jr., and R. W. Buchanan, "The relationship of clozapine and haloperidol treatment response to prefrontal, hippocampal, and caudate brain volumes," American Journal of Psychiatry, vol. 160, no. 8, pp. 1421-1427, 2003.

[22] R. B. Zipursky, J. Zhang-Wong, E. K. Lambe, G. Bean, and M. Beiser, "MRI correlates of treatment response in first episode psychosis," Schizophrenia Research, vol. 30, no. 1, pp. 81-90, 1998.

[23] D. M. Gardner, A. L. Murphy, H. O’Donnell, F. Centorrino, and R. J. Baldessarini, "International consensus study of antipsychotic dosing," The American Journal of Psychiatry, vol. 167, no. 6, pp. 686-693, 2010.

[24] N. Tzourio-Mazoyer, B. Landeau, D. Papathanassiou et al., "Automated anatomical labeling of activations in SPM using a macroscopic anatomical parcellation of the MNI MRI singlesubject brain," NeuroImage, vol. 15, no. 1, pp. 273-289, 2002.

[25] R. Roncone, J. Ventura, M. Impallomeni et al., "Reliability of an Italian standardized and expanded Brief Psychiatric Rating Scale (BPRS 4.0) in raters with high vs. low clinical experience," Acta Psychiatrica Scandinavica, vol. 100, no. 3, pp. 229-236, 1999.

[26] O. Kalla, J. Aaltonen, J. Wahl-ström, V. Lehtinen, I. García Cabeza, and M. González De Chávez, "Duration of untreated psychosis and its correlates first-episode psychosis in Finland and Spain," Acta Psychiatrica Scandinavica, vol. 106, no. 4, pp. 265-275, 2002.

[27] NICE, Schizophrenia: Core Interventions in the Treatment and Management of Schizophrenia in Primary and Secondary Care, National Institute for Health and Clinical Excellence, London, UK, 2002.

[28] NICE, Schizophrenia; Full National Clinical Guidelines on Core Interventions in Primary and Secondary Care, National Institute for Health and Clinical Excellence, London, UK, 2003.
[29] D. I. Velligan, Y. F. Lam, D. C. Glahn et al., "Defining and assessing adherence to oral antipsychotics: a review of the literature," Schizophrenia Bulletin, vol. 32, no. 4, pp. 724-742, 2006.

[30] J. Ventura, K. H. Nuechterlein, K. L. Subotnik, D. Gutkind, and E. A. Gilbert, "Symptom dimensions in recent-onset schizophrenia and mania: a principal components analysis of the 24-item Brief Psychiatric Rating Scale," Psychiatry Research, vol. 97, no. 2-3, pp. 129-135, 2000.

[31] M. G. Harris, L. P. Henry, S. M. Harrigan et al., “The relationship between duration of untreated psychosis and outcome: an eightyear prospective study," Schizophrenia Research, vol. 79, no. 1, pp. 85-93, 2005.

[32] J. P. Lyne, A. Kinsella, and B. O’Donoghue, "Can we combine symptom scales for collaborative research projects?" Journal of Psychiatric Research, vol. 46, no. 2, pp. 233-238, 2012.

[33] J. Welham, T. Stedman, and A. Clair, "Choosing negative symptom instruments: issues of representation and redundancy," Psychiatry Research, vol. 87, no. 1, pp. 47-56, 1999.

[34] S. L. Eckert, P. M. Diamond, A. L. Miller, D. I. Velligan, L. G. Funderburg, and J. E. True, "A comparison of instrument sensitivity to negative symptom change," Psychiatry Research, vol. 63, no. 1, pp. 67-75, 1996.

[35] J. Ashburner and K. J. Friston, "Unified segmentation," NeuroImage, vol. 26, no. 3, pp. 839-851, 2005.

[36] J. Ashburner and K. J. Friston, "Nonlinear spatial normalization using basis functions," Human Brain Mapping, vol. 7, no. 4, pp. 254-266, 1999.

[37] C. D. Good, I. S. Johnsrude, J. Ashburner, R. N. A. Henson, K. J. Friston, and R. S. J. Frackowiak, "A voxel-based morphometric study of ageing in 465 normal adult human brains," NeuroImage, vol. 14, no. 1, part 1, pp. 21-36, 2001.

[38] J. Ashburner and K. J. Friston, "Voxel-based morphometry-the methods," NeuroImage, vol. 11, no. 6, part 1, pp. 805-821, 2000.

[39] E. T. Bullmore, J. Suckling, S. Overmeyer, S. Rabe-Hesketh, E. Taylor, and M. J. Brammer, "Global, voxel, and cluster tests, by theory and permutation, for a difference between two groups of structural MR images of the brain," IEEE Transactions on Medical Imaging, vol. 18, no. 1, pp. 32-42, 1999.

[40] J. Suckling and E. Bullmore, "Permutation tests for factorially designed neuroimaging experiments," Human Brain Mapping, vol. 22, no. 3, pp. 193-205, 2004.

[41] C. Pantelis, D. Velakoulis, P. D. McGorry et al., "Neuroanatomical abnormalities before and after onset of psychosis: a crosssectional and longitudinal MRI comparison," The Lancet, vol. 361, no. 9354, pp. 281-288, 2003.

[42] B. Turetsky, P. E. Cowell, R. C. Gur, R. I. Grossman, D. L. Shtasel, and R. E. Gur, "Frontal and temporal lobe brain volumes in schizophrenia: relationship to symptoms and clinical subtype," Archives of General Psychiatry, vol. 52, no. 12, pp. 1061-1070, 1995.

[43] G. Okugawa, G. C. Sedvall, and I. Agartz, "Reduced grey and white matter volumes in the temporal lobe of male patients with chronic schizophrenia," European Archives of Psychiatry and Clinical Neuroscience, vol. 252, no. 3, pp. 120-123, 2002.

[44] D. C. Glahn, A. R. Laird, I. Ellison-Wright et al., "Metaanalysis of gray matter anomalies in schizophrenia: application of anatomic likelihood estimation and network analysis," Biological Psychiatry, vol. 64, no. 9, pp. 774-781, 2008.

[45] V. Molina, C. Martín, A. Ballesteros, A. G. S. De Herrera, and J. A. Hernández-Tamames, "Optimized voxel brain morphometry: association between brain volumes and the response to 
atypical antipsychotics," European Archives of Psychiatry and Clinical Neuroscience, vol. 261, no. 6, pp. 407-416, 2011.

[46] V. Molina, S. Reig, J. Sanz et al., "Increase in gray matter and decrease in white matter volumes in the cortex during treatment with atypical neuroleptics in schizophrenia," Schizophrenia Research, vol. 80, no. 1, pp. 61-71, 2005.

[47] E. Stip, A. Mancini-Marie, G. Letourneau et al., "Increased grey matter densities in schizophrenia patients with negative symptoms after treatment with quetiapine: a voxel-based morphometry study," International Clinical Psychopharmacology, vol. 24, no. 1, pp. 34-41, 2009.

[48] L. Tomelleri, J. Jogia, C. Perlini et al., "Brain structural changes associated with chronicity and antipsychotic treatment in schizophrenia," European Neuropsychopharmacology, vol. 19, no. 12, pp. 835-840, 2009.

[49] N. E. M. Van Haren, H. E. Hulshoff Pol, H. G. Schnack et al., "Focal gray matter changes in schizophrenia across the course of the illness: a 5-year follow-up study," Neuropsychopharmacology, vol. 32, no. 10, pp. 2057-2066, 2007.

[50] V. Molina, J. A. Hernández, J. Sanz et al., "Subcortical and cortical gray matter differences between Kraepelinian and nonKraepelinian schizophrenia patients identified using voxelbased morphometry," Psychiatry Research, vol. 184, no. 1, pp. 1622, 2010.

[51] J. L. Knoll IV, D. L. Garver, J. E. Ramberg, S. J. Kingsbury, D. Croissant, and B. McDermott, "Heterogeneity of the psychoses: is there a neurodegenerative psychosis?" Schizophrenia Bulletin, vol. 24, no. 3, pp. 365-379, 1998.

[52] I. Nenadic, H. Sauer, and C. Gaser, "Distinct pattern of brain structural deficits in subsyndromes of schizophrenia delineated by psychopathology," NeuroImage, vol. 49, no. 2, pp. 1153-1160, 2010.

[53] T. E. Bakken, C. S. Bloss, J. C. Roddey et al., "Association of genetic variants on $15 \mathrm{q} 12$ with cortical thickness and cognition in schizophrenia," Archives of General Psychiatry, vol. 68, no. 8, pp. 781-790, 2011.

[54] V. Oertel-Knöchel, C. Knöchel, A. Rotarska-Jagiela et al., "Association between psychotic symptoms and cortical thickness reduction across the schizophrenia spectrum," Cerebral Cortex, vol. 23, no. 1, pp. 61-70, 2013.

[55] M. Leung, C. Cheung, K. Yu et al., "Gray matter in first-episode schizophrenia before and after antipsychotic drug treatment: anatomical likelihood estimation meta-analyses with sample size weighting," Schizophrenia Bulletin, vol. 37, no. 1, pp. 199-211, 2011.

[56] R. Smieskova, P. Fusar-Poli, P. Allen et al., "The effects of antipsychotics on the brain: what have we learnt from structural imaging of schizophrenia?-a systematic review," Current Pharmaceutical Design, vol. 15, no. 22, pp. 2535-2549, 2009.

[57] S. Navari and P. Dazzan, "Do antipsychotic drugs affect brain structure? A systematic and critical review of MRI findings," Psychological Medicine, vol. 39, no. 11, pp. 1763-1777, 2009.

[58] N. E. M. Van Haren, H. G. Schnack, W. Cahn et al., "Changes in cortical thickness during the course of illness in schizophrenia," Archives of General Psychiatry, vol. 68, no. 9, pp. 871-880, 2011.

[59] B. Ho, N. C. Andreasen, S. Ziebell, R. Pierson, and V. Magnotta, "Long-term antipsychotic treatment and brain volumes: a longitudinal study of first-episode schizophrenia," Archives of General Psychiatry, vol. 68, no. 2, pp. 128-137, 2011.

[60] N. C. Andreasen, P. Nopoulos, V. Magnotta, R. Pierson, S. Ziebell, and B. Ho, "Progressive brain change in schizophrenia: a prospective longitudinal study of first-episode schizophrenia," Biological Psychiatry, vol. 70, no. 7, pp. 672-679, 2011.

[61] W. Poser, S. Poser, D. Roscher, and A. Argyrakis, "Do benzodiazepines cause cerebral atrophy?" The Lancet, vol. 1, no. 8326, p. 715, 1983

[62] R. S. N. Liu, L. Lemieux, G. S. Bell et al., "Cerebral damage in epilepsy: a population-based longitudinal quantitative MRI study," Epilepsia, vol. 46, no. 9, pp. 1482-1494, 2005.

[63] D. G. Robinson, M. G. Woerner, J. M. J. Alvir et al., "Predictors of treatment response from a first episode of schizophrenia or schizoaffective disorder," The American Journal of Psychiatry, vol. 156, no. 4, pp. 544-549, 1999.

[64] J. Peuskens, "The evolving definition of treatment resistance," Journal of Clinical Psychiatry, vol. 60, no. 12, pp. 4-8, 1999. 


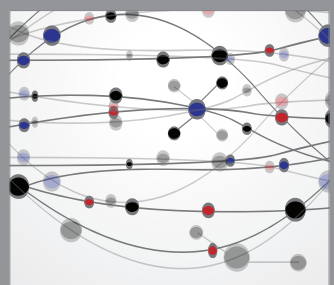

The Scientific World Journal
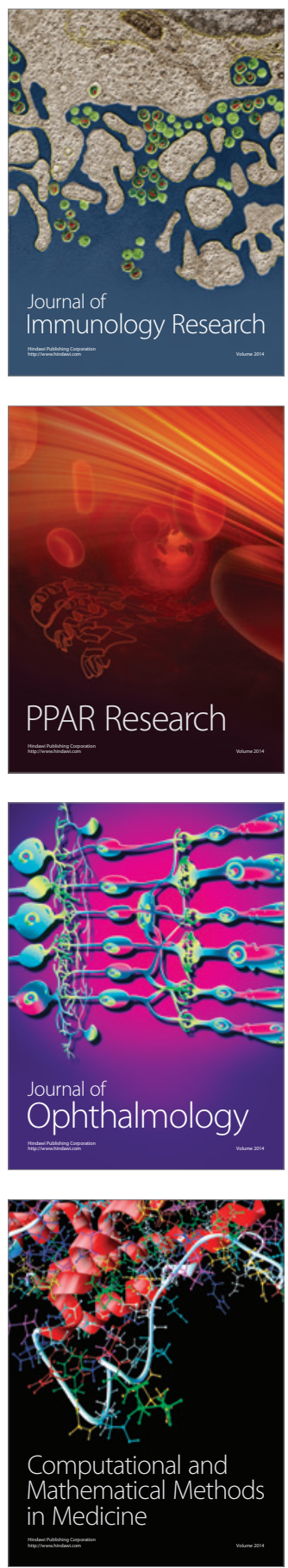

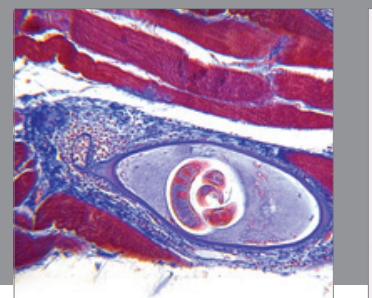

Gastroenterology

Research and Practice
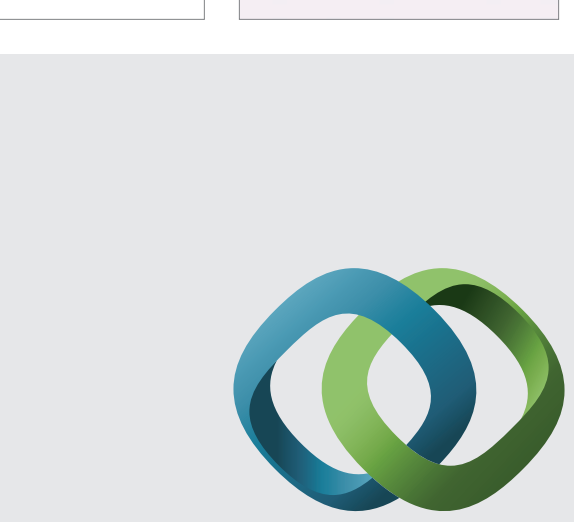

\section{Hindawi}

Submit your manuscripts at

http://www.hindawi.com
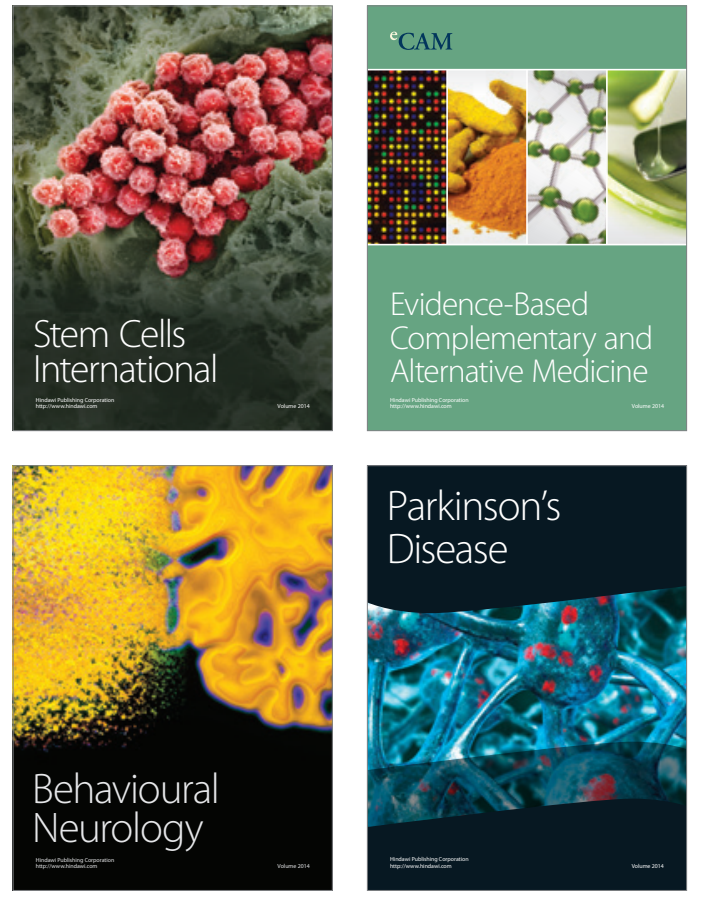


Disease Markers
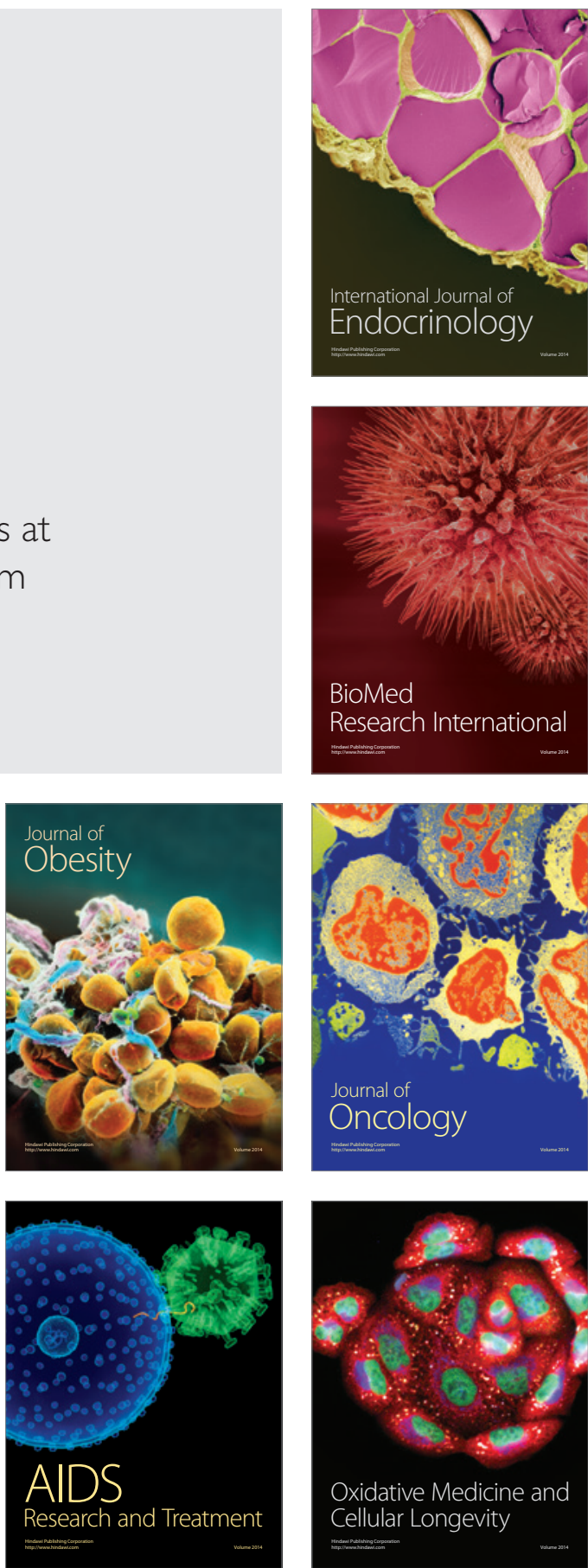